\title{
Picosecond cyclization reaction dynamics of furan-based diarylethene with thiosemicarbazone side-chain groups
}

\author{
A. A. Khodko ${ }^{\text {a }}$, V. V. Khomenko ${ }^{\text {, }}$ O. D. Mamuta ${ }^{a}$, lu. P. Mukha ${ }^{\text {b }, ~ D . ~ O . ~ S y s o i e v ', ~ T . ~ H u h n c, ~}$ \\ S. V. Snegir ${ }^{b}$, and N. M. Kachalova ${ }^{a}$

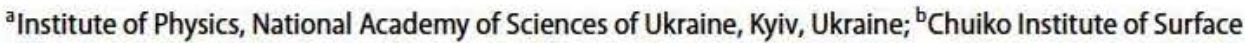 \\ Chemistry, National Academy of Sciences of Ukraine, Kyiv, Ukraine; ' University of Konstanz, Konstanz, Germany
}

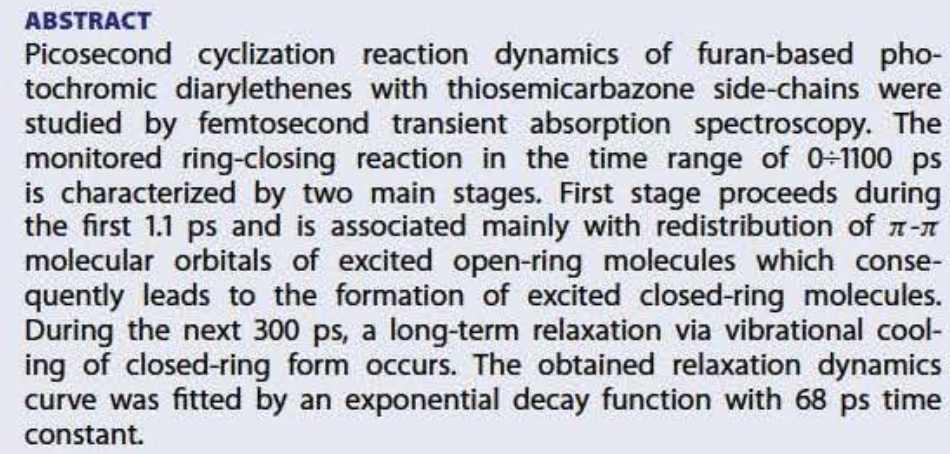

\section{Introduction}

Photochromism is a photoinduced reversible chemical reaction, during which materials change their optical, electrical and conformational properties [1]. Among known photochromic molecules, the diarylethenes (DAEs) can be reversely switched between two welldefined open-ring (OF) and closed-ring (CF) forms by irradiation with ultraviolet/visible light and/or by electrical stimulation. This type of switchers attracts much attention for the last few years $[2,3]$. Moreover, these molecules can be contacted in two-wire geometry making them applicable as organic elements in optoelectronics [4].

For implementation into real molecular electronics devices such as logic gates [5] and optically switchable transistors $[6,7]$ both $\mathrm{OF}$ and $\mathrm{CF}$ of DAEs should possess the key properties [1]: thermal stability, high fatigue resistance, rapid optical response, and minor structural change while switching. Most of these requirements are fulfilled, if appropriate photochromic core and side-chain groups are combined $[2,8]$. Among the known photochromic molecules, DAEs display optically-induced reversible switching in solutions in the femtosecond regime [9-18]. Furthermore, while DAEs being attached to metallic electrodes at least ring-closing reaction can be achieved $[19,20]$. Unfortunately, the suitable side-chain groups that can be used to link the photochromic core with metal electrodes for further construction of a reversible switchable metal-organic device still were not identified even though numerous

CONTACT A.A. Khodko khodkoalina@gmail.com Institute of Physics, NAS of Ukraine, 46 Nauky Pros., Kyiv-068, Ukraine. 

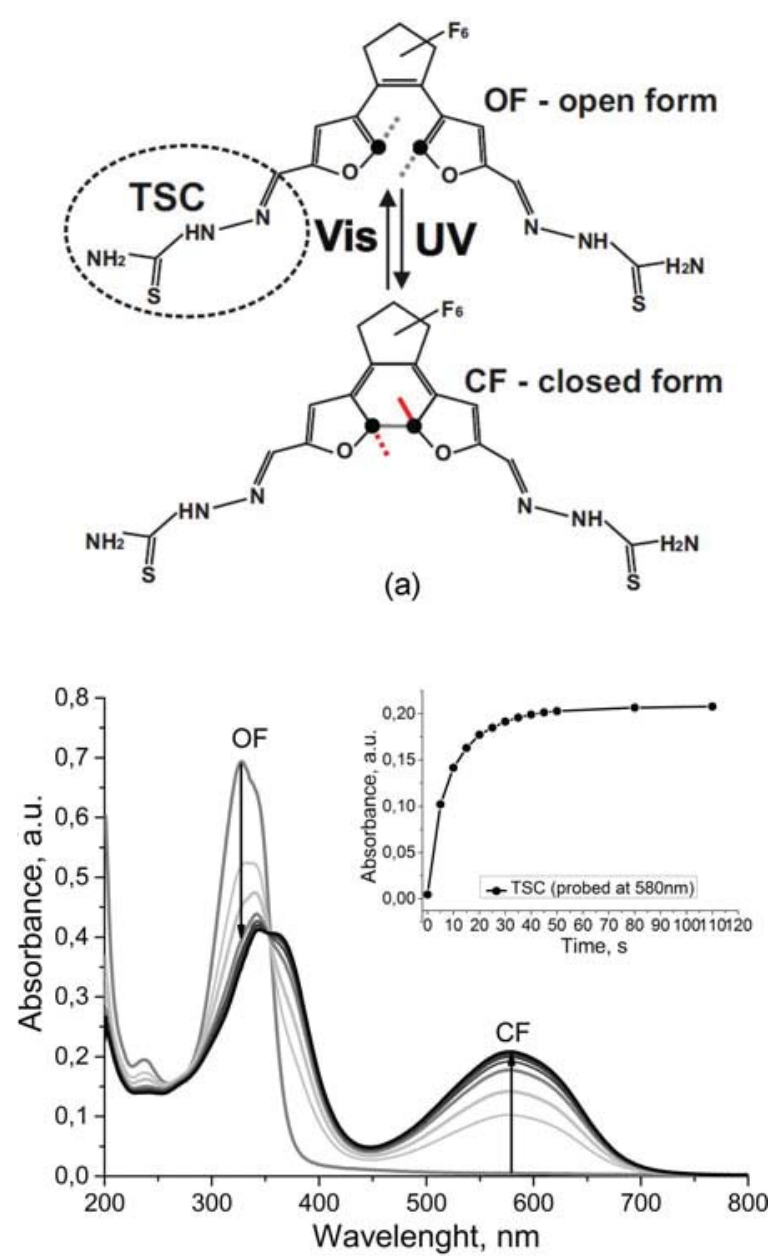

(b)

Figure 1. (a) Structures of $\mathbf{O F}$ and $\mathbf{C F}$ of CF5-TSC. The CF can be obtained under irradiation of ultraviolet (UV) light while reverse reaction occurs under visible (vis) light irradiation. (b) Photoinduced changes in absorbance spectra of OF and CF under UV illumination $\left(365 \mathrm{~nm}, 15 \mathrm{~mW} / \mathrm{cm}^{2}\right)$; the insert shows absorbance changes tracked at $580 \mathrm{~nm}$ in time scale $0 \div 110 \mathrm{sec}$.

functionalized DAEs were synthesized and discussed in the literature $[2,7]$. One of the most successful structures so far is a design by Feringa's group based on a thiophene switching core, attached via a cross-conjugated meta-phenyl linking group and equipped with gold anchoring groups for molecular grafting onto $\mathrm{Au}(111)$ surface [21] and for binding to gold nanoparticles [22]. By replacing the thiophene in the photochromic core with its oxygen homologue furan bearing DAE were accessible. Their conductance on a single-molecule level was investigated recently by break-junction experiments at low temperatures [23]. Moreover, the aromatic linkers were replaced by partly conjugated heteroatom-rich groups efficiently decoupling the molecular switch from the gold electrodes and, thus, avoiding unintended quenching of the exited state [24].

Using this design, a new type of furan-based DAE, the 1,2-bis(2-methyl-5-((E)-(2thiocarbamoylhydrazono)methyl]-furan-3-yl)-perfluorocyclopentene (CF5-TSC) was synthetized and purified according to [24]. The thiosemicarbazone (TSC) side-chain [25] was used to connect the molecule successfully with gold electrodes [26]. Figure 1a represents 
photoinduced ring-closing and ring-opening (a) of CF5-TSC under consecutive UV/vis light irradiation. The formation of the closed form (CF) is characterized in absorption spectra (Figure 1b) by the appearance of a new broad absorption band in the range of $450 \div 730 \mathrm{~nm}$ and a decrease of absorption of the bands in the shorter wavelength range of $250 \div 450 \mathrm{~nm}$. The reverse ring-opening reaction can be induced by visible light which leads to restoring of initial spectra of $\mathbf{O F}$ without absorption band in the visible light region.

Both CF and OF are thermally stable, reversibly switchable and have appropriate optical properties (photoconversion quantum yields: cyclization -0.28 , cycloreversion $-4.9 \cdot 10^{-3}$ ) [25], This makes molecules attractive candidates for further applications in the molecularscaled flexible devices with optically modulated properties [4]. However, to use them in a construction of devices with a rapid optical response the dynamics of ring-closing and opening reactions have to be studied. This will allow not only to define the time ranges, during which these reactions occur, but clarify possible operation time limits of the devices as well. Consequently, we continue study of the optical properties of CF5-TSC and particularly will pay attention to the ring-closing reaction, using femtosecond pump-probe spectroscopy. We expect that cyclization reaction of CF5-TSC will be a complicated combination of sequential processes on ultrafast timescale: from hundreds of femtoseconds up to tens of picoseconds as for DAEs with similar thiophene-based photochromic core [10]. If monitored ring-closing reaction of the CF5-TSC will occur similarly to the thiophene-based DAEs it will mean that oxygen substituting in the photochromic core has no influence on the ring-closing time range compared to thiophene-based DAEs [9-16].

\section{Experimental}

The photoinduced dynamics were explored by femtosecond transient absorption spectroscopy at the Center for collective use "Femtosecond Laser Complex" at the Institute of Physics (Kyiv, Ukraine) $[27,28]$. The amplified femtosecond pulses ( $\mathrm{E}=0.5 \mathrm{~mJ}, \tau=150 \mathrm{fs}$, $\lambda=800 \mathrm{~nm}, v=1 \mathrm{kHz}$ ) from Ti:Sapphire laser (Coherent, USA) were split into two channels: "pump" initiated cyclization reaction, while probe tracked the ultrafast changes in absorption, which have occurred in the sample. Since cyclization occurs under UV illumination (Figure $1 \mathrm{~b}$ ), we used the third harmonic generation ( $\mathrm{E}=0.4 \mathrm{uJ}, \mathrm{t}=150 \mathrm{fs}, \lambda=266 \mathrm{~nm}, v=$ $250 \mathrm{~Hz}$ ), obtained via the femtokit for third harmonic generation (Eksma Optics). As probe pulse white light supercontinuum generation (SC) $-\lambda=540 \div 600 \mathrm{~nm}$, obtained in $\mathrm{Al}_{2} \mathrm{O}_{3}$ crystal by femtosecond pulses at $\lambda=800 \mathrm{~nm}$ was used. The spectral range of probing was chosen in accordance to the CF absorption band under stationary UV/vis illumination (Figure 1b). The probe beam was split into signal and idler beams before crossing the sample, then both beams were recorded by multichannel spectrograph (Imaging Spectrograph SP-2500i, Acton, USA). To correct SC fluctuations, the transient absorbance spectra were obtained by comparing signal and idler spectra for different time delays, and then, the group-velocity dispersion was corrected at all obtained transient traces [29].

Ethanolic solution of CF5-TSC with optimal concentration $\mathrm{C}=5 \cdot 10^{-5} \mathrm{~mol} \cdot \mathrm{L}^{-1}$ was used. The CF5-TSC solution was pumped through a quartz flow cell with $2 \mathrm{~mm}$ optical path using a peristaltic pump with flow rate, $\mathrm{Q}=2 \mathrm{ml} / \mathrm{s}$. To avoid accumulation of $\mathrm{CF}$, we irradiated the solution in the buffer volume by broad visible radiation (Schott KL $1500 \mathrm{LCD}, \mathrm{P}=150 \mathrm{~W}$ ), which caused the reversible ring-opening reaction. All studies were performed at room temperature $\mathrm{T}=22 \pm 0.2^{\circ} \mathrm{C}$. All solutions of CF5-TSC were stored in the dark to avoid 


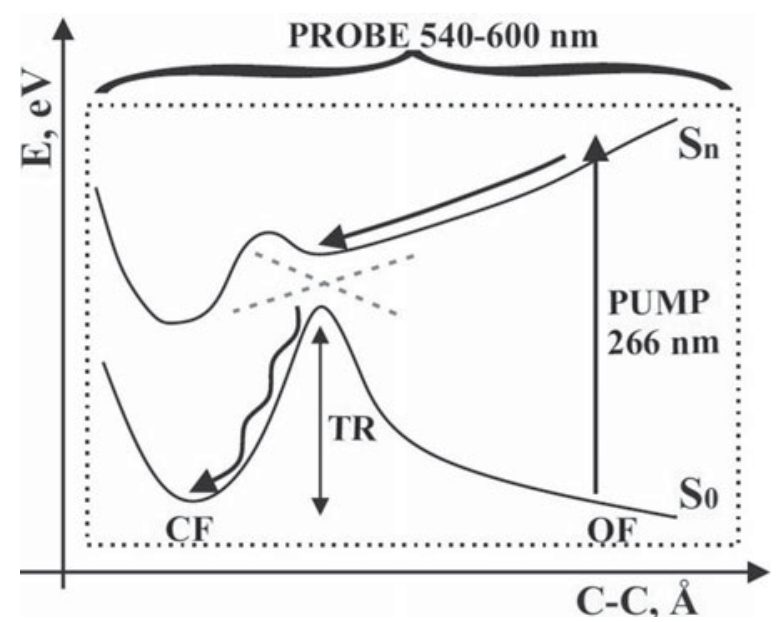

Figure 2. Schematic representation of transition from OF to CF during the cyclization process. TR - transitional state, $\mathrm{S}_{0}$ and $\mathrm{S}_{\mathrm{n}}$ are the ground and exited state of the molecules, respectively.

uncontrolled photochromic reactions which may occur under the action of natural or/and artificial light.

\section{Results and discussion}

The photoinduced changes in optical density of photochromic molecules with TSC side chains were studied by pump-probe measurements using femtosecond UV pump pulses ( $\lambda=$ $266 \mathrm{~nm})$ and SC probe pulses $(\lambda=540 \div 600 \mathrm{~nm})$ at a variable temporal delay. We monitored the transition from $\mathbf{O F}$ to $\mathbf{C F}$ in two separate time ranges. The first stage is associated mainly with ring-closing reaction (Figure 1a). This stage was studied in a time range from $0 \div 3 \mathrm{ps}$ (Figure 3). The further long-term relaxation (Figure 2) [10] of exited CF after the process of $\mathrm{C}$-C bond formation was monitored in the range of $0 \div 1100$ ps (Figure 4 ).

At the beginning of the first stage each pump pulse initiates the cyclization reaction: $\mathbf{O F}$ molecules absorb UV light and move to a higher excited state $S_{n}$ (Figure 2). After a fast electronic state mixing, an electron density is redistributed. In a consequence, the excited $\mathbf{O F}$ is passing thought the conical intersection [11] to the transitional state (TR) between the $\mathbf{O F}$ and CF ground states. The further formation of C-C bond occurs following by $\pi-\pi$ orbital coupling in the photochromic core at TR (i.e., appearance of CF molecules).

These consistent processes are tracked by a probe pulse, since the growth of optical density of $\mathbf{O F}$ starts at $0 \mathrm{ps}$ and finishes near $1.6 \mathrm{ps}$ (Figure 3).

The gradual changes of optical density in the current experiment was monitored in the range of $\lambda=540 \div 600 \mathrm{~nm}$ (Figure 3a) according to the position of CF maximum (Figure 1b), that appears during photoinduced cyclization reaction. This is expected since freshly formed $\mathrm{CF}$ molecules absorb light in this range (Figure 1b). From the obtained data we plotted time profile of the optical spectra changes at $580 \mathrm{~nm}$ (Figure 3b). This plot has nonlinear behavior reflecting the dynamics of ring-closing reactions. To characterize the gradual growth of the optical density, we determine the rise time as the time were absorbance changes from $10 \%$ to $90 \%$ of the final value of optical density. We found the upper boundary of the rise time is close to $1.1 \mathrm{ps}$ (Figure 3b). Another approach to characterize this process is fitting of the experimental data with convolution of exponential function and instrument response function (Figure $3 b)$. For the minimal expected instrument response function (FWHM $=212 \mathrm{fs}$ ), we obtained 


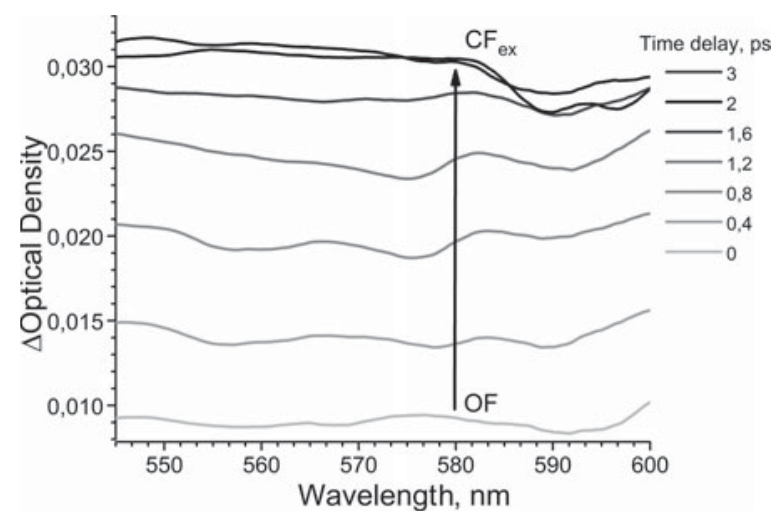

(a)

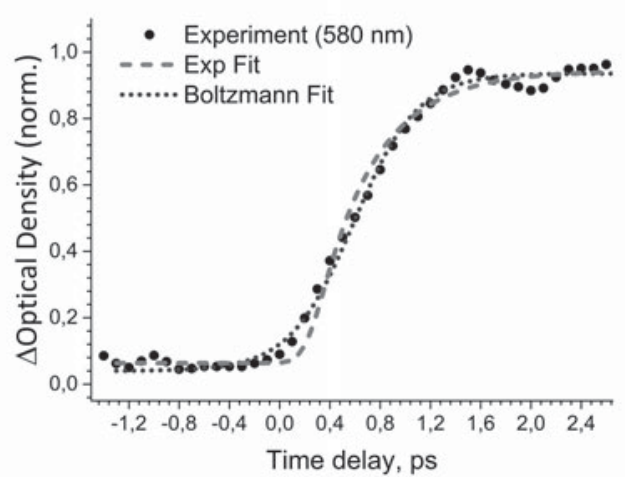

(b)

Figure 3. Photoinduced changing of optical density during transition of $\mathbf{O F}$ to excited closed-ring molecules $\left(\mathbf{C F}_{\text {ex }}\right)$, initiated by femtosecond pump pulses at $\lambda=266 \mathrm{~nm}$ and probed at $\lambda=540 \div 600 \mathrm{~nm}$ : (a) Timeresolved spectra in the time range $0 \div 3 \mathrm{ps}$; (b) Normalized time profile of ring-closing reaction obtained by the monitoring of optical density growth at $580 \mathrm{~nm}$ was fitted by sigmoidal and exponential functions [30].

an upper boundary of the time constant of 0.49 ps. Further measurements with shorter laser pulses are required for a more precise determination of the ring-closing time constant. We do not exclude that in solvents with different polarity CF5-TSC might display different time of cyclization reaction $[15,35]$.

The next stage was associated with further long-term relaxation processes of $\mathbf{C F}_{\mathbf{e x}}$ of the newly formed molecules (Figure 2). They are characterized by vibrational cooling of a hot CF species to the thermal equilibrium (Figure 4). After 3 ps delay a noticeable decrease of the optical density was detected that corresponds to the further relaxation of $\mathbf{C F}_{\mathbf{e x}}$.

After 300 ps, the optical density level is stabilized, which means that the relaxation process is finished. The relaxation time profile was fitted by exponential decay function with time constant, $\mathrm{t}_{\text {relax }}=68 \pm 15 \mathrm{ps}$, in line with the proposed reaction model [10] with similar dynamics compared to other DAEs $[11,12]$. Thus, we found that the upper boundary of the rise time is 1.1 ps for photoinduced cyclization reaction of CF5-TSC. The determined time is slightly larger than the one for some others similar thiophene-based DAEs [10-13], but is lower compared to equivalent DAEs with various modifications of side-chains groups [14-16]. Therefore, we suspect that exactly the side-chain groups of the molecules play the key role in processes of ring-closing reaction $[31,32]$. This might be explained by the difference in the nature of these groups, since some can withdraw electron density from the photochromic core, while others can donate electron density $[33,34]$. Therefore, the cyclization reaction 


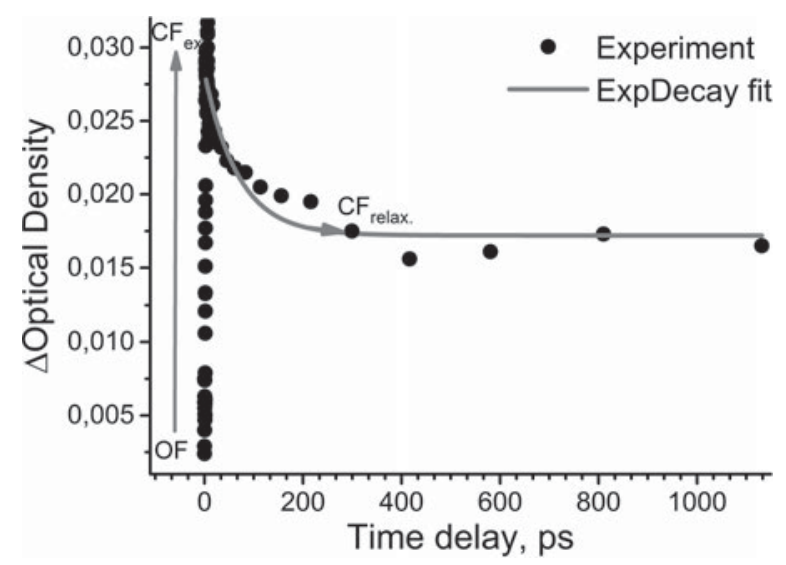

Figure 4. Complete time profile of $\mathbf{C F}$ formation is displaying an optical density change from 0 to $1100 \mathrm{ps:}$ C-C bond formation ( $0 \div 1.1 \mathrm{ps})$ discussed above and further relaxation of $\mathbf{C F}_{\mathrm{ex}}$ of $\mathbf{C 5 F}^{-T S C}(3 \div 300 \mathrm{ps})$. The $\mathrm{x}$-axis represents $\mathrm{C}-\mathrm{C}$ distance of newly formed bond during photo cyclization.

dynamics, which involves the process of $\pi-\pi$ molecular orbitals (MO) redistribution within the photochromic core, may have quite different time scale. Moreover, such reaction may be sensitive to the pump wavelength [18] and solvent polarity [15, 35].

The other quite important finding of our work is the study of the substituent effect of oxygen atoms within a structure of CF5 photochromic core. Taking into account that the determined cyclization time of CF5-TSC is close to all CF5-based DAEs studied by pump-probe spectroscopy $[10,12,16]$ and the relaxation profile has a similar shape $[9,11,13]$, we conclude that the exchange of thiophene against furan apparently has no impact on the ring-closing dynamics of the resulting DAE. Hence, for an application of CF5-based DAE in a combination with gold electrodes, the use of furan-based DAE will be a technical benefit, since oxygen atoms are chemically inert towards gold when compared with sulfur [25].

\section{Conclusions}

The picosecond cyclization and further relaxation dynamics of a new sulfur-free furan-based DAE with functional TSC side chains were studied by femtosecond transient absorption spectroscopy in detail. We found that the upper boundary of the rise time is $1.1 \mathrm{ps}$ for the cyclization of C5F-TSC, in which the sulfur atoms in the photochromic core were replaced by oxygen. This is equivalent value to the cyclization time range of similar thiophene-based molecules. After $\pi-\pi$ orbital coupling, the long-term relaxation to thermal equilibrium of $\mathbf{C F}$ was detected for C5F-TSC molecules. Therefore, the femtosecond pump-probe technique is a suitable tool to systematically study the correlation of structural modifications of DAEs and their optical properties on the ultrafast time scale aiming to develop further functionalization strategies.

\section{Acknowledgments}

The work was supported by the Grant of NAS of Ukraine for young scientists for realization of the research project, 2015-2016. Authors are grateful to the staff of the Center for collective use Femtosecond Laser Complex for useful discussions and measurements, and to Dr. D. Brida from the University of Konstanz for precise consultations on further experiments. Syntheses were performed by D.S. at University of Konstanz, D.S. was supported by DFG project Hu 1682/3-1. 


\section{References}

[1] Irie, M. (2010). Proc. Jpn. Acad., Ser. B, 472-483.

[2] Irie, M., Fukaminato, T., Matsuda, K., \& Kobatake, S. (2014). Chem. Rev., 114, 12174-12277.

[3] Abendroth, J. M., Bushuyev, O. S., Weiss, P. S., \& Barrett, C. J. (2015). ACS Nano, 9, 7746-7768.

[4] Kim, D., Jeong, H., Lee, H., Hwang, W. T., Wolf, J., Scheer, E., Huhn, T., Jeong, H., \& Lee, T. (2014). Adv. Mater., 26, 3968-3973.

[5] Wu, Y., Guo, Z., Zhu, W.-H., Wan, W., Zhang, J., Li, W., Li, X., Tian, H., \& Li, A. D. Q. (2016). Mater. Horiz., 3, 124-129.

[6] Hayakawa, R., Higashiguchi, K., Matsuda, K., Chikyow, T., \& Wakayama, Y. (2013). ACS Appl. Mater. Interfaces, 5, 3625-3630.

[7] Gemayel, M. E., Börjesson, K., Herder, M., Duong, D. T., Hutchison, J. A., Ruzié, C., Schweicher, G., Salleo, A., Geerts, Y., Hecht, S., Orgiu, E., \& Samorì, P. (2015). Nat. Commun., 6, 1-8.

[8] Irie, M. (2010). Photochem. Photobiol. Sci., 9, 1535-1542.

[9] Tamai, N., \& Miyasaka, H. (2000). Chem. Rev., 100, 1875-1890.

[10] Hania, P. R., Telesca, R., Lucas, L. N., Pugzlys, A., van Esch, J., Feringa, B. L., Snijders, J. G., \& Duppen, K. (2002). J. Phys. Chem. A, 106, 8498-8507.

[11] Hania, P. R., Pugzlys, A., Lucas, L. N., de Jong, J. J. D., Feringa, B. L., van Esch, J. H., Jonkman, H. T., \& Duppen, K. (2005). J. Phys. Chem. A, 109, 9437-9442.

[12] Shim, S., Eom, I., Joo, T., Kim, E., \& Kim, K. S. (2007). J. Phys. Chem. A, 111(37), 8910-8917.

[13] Pontecorvo, E., Ferrante, C., Elles, C. G., \& Scopigno, T. (2014). J. Phys. Chem. B, 118(24), 69156921.

[14] Murata, R., Yago, T., \& Wakasa, M. (2016). J. Phys. Chem. A, 119(45), 11138-11145.

[15] Ishibashi, Y., Umesato, T., Fujiwara, M., Une, K., Yoneda, Y., Sotome, H., Katayama, T., Kobatake, S., Asahi, T., Irie, M., \& Miyasaka, H. (2016). J. Am. Chem. Soc., 120(2), 1170-1177.

[16] Bens, A. T., Ern, J., Kuldova, K., Trommsdorff, H. P., \& Kryschi, C. (2002). J. Lumin., 94, 51-54.

[17] Buckup, T., Sarter, C., Volpp, H.-R., Jäschke, A., \& Motzkus, M. (2015). J. Phys. Chem. Let., 6, 4717-4721.

[18] Aloïse, S., Sliwa, M., Pawlowska, Z., Réhault, J., Dubois, J., Poizat, O., Buntinx, G., Perrier, A., Maurel, F., Yamaguchi, S., \& Takeshita, M. (2010). J. Am. Chem. Soc., 132, 7379-7390.

[19] Kim, D., Jeong, H., Lee, H., Hwang, W. T., Wolf, J., Scheer, E., Huhn, T., Jeong, H., \& Lee, T. (2014). Adv. Mater., 26, 3968-3973.

[20] Dulić, D., van der Molen, S. J., Kudernac, T., Jonkman, H. T., de Jong, J. J. D., Bowden, T. N., van Esch, J., Feringa, B. L., \& van Wees, B. J. (2003). Phys. Rev. Lett., 91, 207402.

[21] Katsonis, N., Kudernac, T., Walko, M., van der Molen, S. J., van Wees, B. J., \& Feringa, B. L. (2006). Adv.Mater., 18, 1397-1400.

[22] van der Molen, S. J., Liao, J., Kudernac, T., Agustsson, J. S., Bernard, L., Calame, M., van Wees, B. J., Feringa, B. L., \& Schönenberger, C. (2009). Nano Lett., 9, 76-80.

[23] Kim, Y., Hellmuth, T. J., Sysoiev, D., Pauly, F., Pietsch, T., Wolf, J., Erbe, A., Huhn, T., Groth, U., Steiner, U. E., \& Scheer, E. (2012). Nano Lett., 12, 3736-3742.

[24] Sysoiev, D., Yushchenko, T., Scheer, E., Groth, U., Steiner, U. E., Exner, T. E., \& Huhn, T. (2012). Chem. Commun., 48, 11355-11357.

[25] Sysoiev, D., Fedoseev, A., Kim, Y., Exner, T. E., Boneberg, J., Huhn, T., Leiderer, P., Scheer, E., Groth, U., \& Steiner, U. E. (2011). Chemistry - A European Journal, 17, 6663-6672.

[26] Snegir, S., Mukha, I., Sysoiev, D., Lacaze, E., Huhn, T., \& Pluchery, O. (2016). Materialwiss. Werkstofftech., 47, 229-236.

[27] Blonsky, I. V., Dmitruk, I. M., Zubrilin, M. G., Kadan, V. M., Korenyuk, P. I., Pavlov, I. A., \& Salnikov, V. O. (2008). Nanosystems, 6, 45-74.

[28] Khodko, A. A., Snegir, S. V., Khomenko, V. V., Mamuta, O. D., Voitsechovych, V. S., \& Kachalova, N. M. (2015). Proc. Ukr. Acad., 12, 57-63.

[29] Ziolek, M., Naskrecki, R., \& Karolczak, J. (2004). Opt. Commun., 241, 221-229.

[30] Spörkel, L., Jankowska, J., \& Thiel, W. (2015). J. Phys. Chem. B, 119, 2702-2710.

[31] Pu, S., Zheng, C., Le, Z., Liu, G., \& Fan, C. (2008). Tetrahedron, 64, 2576-2585.

[32] Fan, C., Pu, S., Liu, G., \& Yang, T. (2008). J. Photochem. Photobiol. A, 194, 333-343.

[33] Wolf, J., Eberspächer, I., Groth, U., \& Huhn, T. (2013). J. Org. Chem., 78, 8366-8375..

[34] Wolf, J., Huhn, T., \& Steiner, U. E. (2015). Phys. Chem. Chem. Phys., 17, 6066-6075.

[35] Irie, M., \& Sayo, K. (1992). J. Phys. Chem., 96, 7671-7674. 